\title{
ESTRATEGIA TIC PARA ENSENAR LA FUNCIÓN LINEAL EN ESTUDIANTES UNIVERSITARIOS
}

\section{TIC STRATEGY TO TEACH THE LINEAR FUNCTION IN UNIVERSITY STUDENTS}

\author{
Edgardo Escorcia Caballero ${ }^{1}$ \\ Víctor Riveros Villarreal ${ }^{2}$ \\ Rolando Escorcia Caballero ${ }^{3}$ \\ Universidad del Magdalena, Santa Marta, \\ Colombia
}

Universidad del Zulia, Maracaibo, Venezuela

\section{RESUMEN}

La presente investigación enmarcada en el paradigma positivista de naturaleza cuantitativa explicativa y diseño cuasiexperimental tipo pretest-postest, con grupo control, tiene como objetivo estimar el efecto de la implementación del software WINPLOT en el

1 https://orcid.org/0000-0002-4587-5711, eescorcia@unimagdalena.edu.co, Licenciado en Matemática y Física, Especialista en Matemática, Magister Scientiarium en Matemática mención: Docencia. Docente Tiempo Completo Universidad del Magdalena, Santa Marta, Colombia. 2 https://orcid.org/0000-0002-5401-6951, vriveros75@gmail.com, Licenciado en Educación mención: Ciencias Matematicas, Magister en Matemáticas aplicadas, Doctor en Ciencias Humanas. Profesor Titular Universidad del Zulia, Maracaibo, Venezuela.

3 https://orcid.org/0000-0001-7605-2876, reescorcia@unimagdalena.edu.co, Licenciado en Ciencias Sociales, Especialista en Edumática, Magister en Educación con énfasis en Docencia Universitaria, Doctor en Educación. Docente Tiempo Completo Universidad del Magdalena, Santa Marta, Colombia. aprendizaje de la función lineal en estudiantes del primer semestre de la Facultad de Ciencias Empresariales y Económicas de la Universidad del Magdalena. Se definió una muestra conformada por 30 estudiantes, divididos en dos grupos, Experimental y Control, que cursaron la asignatura Cálculo Diferencial en el período 2017 I. La información se recogió a través de dos pruebas (pretest- postest). Los resultados evidencian una correlación fuerte entre las variables objeto de estudio presentándose una mejora significativa en sus calificaciones con relación a las obtenidas antes del experimento. Se concluyó que con la implementación de dicho software se favorece de manera positiva el aprendizaje de los estudiantes, pues se produjo mejora en el desempeño académico de los estudiantes del grupo experimental con respecto 
al grupo control. Se concluye además que la utilización del software WINPLOT, constituye una estrategia didáctica que contribuye a mejorar el aprendizaje de los estudiantes en el tema de la función lineal.

\section{PALABRAS CLAVE}

Software, Enseñanza, Aprendizaje, Función Lineal, Winplot, (Fuente: Tesauro de la Unesco).

\section{ABSTRACT}

The present study corresponds to investigation framed in the positivist paradigm of explanatory quantitative nature and quasi-experimental design pretest-posttest, with control group, the objective is aims to estimate the effect of the implementation of the WINPLOT software on the learning of the linear function in students of the first semester of the Faculty of Business and Economic Sciences of the Universidad del Magdalena. A sample made up of 30 students was defined, divided into two groups, Experimental and Control, who enrolled the Differential Calculus subject in the 2017-I period, and the information was collected through two tests (pretest - posttest). The results show a strong correlation between the variables under study, presenting a significant improvement in their scores in relation to those obtained before the experiment. It was concluded that the implementation of this software positively favors student learning, since there was an improvement in we academic performance in the experimental group compared to control group. It is also concluded that the use of the WINPLOT software constitutes a didactic strategy that contributes to improving student learning on the subject of linear function.

\section{KEY WORDS}

Software, Learning, Linear Function, Winplot.

\section{INTRODUCCIÓN}

El rendimiento académico de los estudiantes constituye un indicador estratégico para las instituciones de Educación Superior, IES, que proporciona una sólida información para la toma de decisiones relacionadas con la definición de políticas institucionales que buscan atender a la población estudiantil con miras a disminuir los índices de "mortalidad académica" y deserción del sistema educativo, el cual, en el país, arroja valores preocupantes tanto a nivel nacional como regional.

En este sentido, Tuero et all (2018), Belloc, Maruotti y Petrella (2011) asumen que si un alumno en el contexto universitario no presenta condiciones adecuadas de estudio tiene altas probabilidades de desertar. Sin embargo, esta no es la única razón del fenómeno de la deserción; variables sociológicas, como la vinculación existente entre los estudiantes y sus profesores (Alzás y Casas, 2015; Bernardo, Cervero, Esteban, Fernández, y Núñez, 2016; así como las económicas y personales también constituyen factores que influyen y pueden conllevar a los estudiantes a desertar. Los estudios de Aguilera y Jiménez, 2012; Bozick, (2007); Elías, (2008); Feixas, Muñóz, Gairín, Jiménez, y Navarro, (2015); García y Adrogué, (2015); Goldenhersh, Coria, y Saino, (2011); Rué, (2014); dan cuenta de ello, y algunos relacionan el abandono de los estudios con vinculación al trabajo debido a los horarios que se cruzan con la asistencia a clases

De igual manera, se encuentra que otro de los determinantes del abandono de los estudiantes a nivel universitario está dado por el bajo rendimiento académico, pues éste y la repetición de cursos provocan dificultades para la institución y para la población estudiantil (Montero et al., 2007), estas variables, son consideradas por el estado colombiano, anualmente, para determinar "la calidad de las 
Instituciones de educación superior" y a partir de estas, sumadas a otras variables, genera un esquema de "incentivos" relacionados con la asignación de recursos adicionales, que, aunque no entran a la base presupuestal, constituyen una bolsa a la cual las universidades oficiales le apuntan y tratan de mejorar sus indicadores año a año.

No obstante, la calidad se relaciona, no sólo con el desempeño de los estudiantes en las disciplinas, sino además con criterios de financiamiento, cualificación docente, procesos evaluativos, innovación, enseñanza y aprendizaje. En este sentido, las prácticas pedagógicas, entendidas como el conjunto de actividades que permiten planificar, desarrollar y evaluar procesos intencionados de enseñanza mediante los cuales se favorece el aprendizaje de contenidos (conocimientos, habilidades, actitudes y valores) por parte de personas que tienen necesidades de formación (Wilson, 1996), constituyen, tal vez, la variable de mayor impacto, y relevancia para favorecer una educacion de alta calidad.

La práctica pedagógica incluye aquellos procesos tendientes a favorecer la formación del ser humano y el aprendizaje de las disciplinas, caracterizada por incluir todas aquellas situaciones que no son, ni accidentales ni casuales, y que requieren, por demás, estar planificadas representando lo que Marcelo (2001) denomina, ambientes de aprendizaje. Enseñar y aprender, por tanto, son dos términos unidos por una sola intención: producir construcción y apropiación de conocimiento y competencia por parte de las personas que deciden implicarse en este juego.

Sin embargo, es frecuente observar en las aulas de las IES de nuestra región, la predominancia de un modelo de enseñanza por transmisión, el cual, tiene su fundamento, Según Calatayud, Gil y Gimeno, (1992) en unas suposiciones inadecuadas: a) Enseñar es una tarea fácil y no requiere una especial preparación (basta el conocimiento de la materia, sentido común y algo de experiencia).

b) El proceso de enseñanza-aprendizaje se reduce a una simple transmisión y recepción de conocimientos.

c) El fracaso de muchos alumnos se debe a sus propias deficiencias: bajo nivel, falta de capacidad, etc.

En este sentido y refiriéndose a la enseñanza de las ciencias, afirman estos autores, que pareciera claro, que las estrategias tradicionales de enseñanza son poco eficaces para promover el aprendizaje significativo. Si se parte de esta premisa, entonces es conveniente buscar alternativas distintas que incluyan el uso de las nuevas tecnologías de la comunicación abandonando la noción de método de enseñanza y cambiarla por estrategias de enseñanza. Las cuales se concretan en actividades en las que "se maneja cierta información procedente de unas determinadas fuentes, mediante procedimientos concretos (asociados a unos medios didácticos) y en relación con unas metas explícitas o implícitas" (García y Cañal, 1995, p. 7). En este último ámbito -la docencia- se viene observando, cómo la integración de las tecnologías digitales por parte de los docentes, está modificando la concepción tradicional de docente y de enseñanza (Marcelo, C., \& Rijo, D., 2019), sin embargo, aún se observa mucha resistencia de parte de profesores hacia el uso de las tecnologías en el desarrollo de las clases y mantienen la utilización de fórmulas que en la mayoría de los casos, los estudiantes deben memorizar. No obstante,

Durante los últimos años, el uso de la tecnología se ha vuelto más frecuente entre las personas por las ventajas y facilidades 
que ofrece. A través de ella se van adquiriendo conocimientos y desarrollando destrezas digitales que utilizamos para nuestro beneficio. Los estudiantes cada día son más conscientes de los procesos que llevan a cabo para organizar y dominar su propio aprendizaje con el uso de las tecnologías. (Marcelo, C., \& Rijo, D., 2019).

Sin embargo, la matemática constituye una de las ciencias, que más ha tardado en incorporar estas estrategias y dar un giro importante hacia la utilización de las TIC como apoyo a los procesos de enseñanza y aprendizaje, siendo frecuente el uso de metodologías tradicionales y la realización de procesos mecánicos, descontextualizados sin reflexiones importantes en los estudiantes sobre la utilidad que tienen los conceptos estudiados en su proceso formativo académico y en la cotidianeidad (Vega, et al. 2016). En este sentido (Carrascosa y Gil, 1985) manifiestan que existe evidencia amplia de que los estudiantes al abordar el análisis de problemas científicos, utilizan estrategias de razonamiento y metodologías superficiales, o aplican "heurísticos importados del contexto cotidiano, pero de dudosa utilidad cuando se trabaja con contenidos científicos" (Pozo, Sanz, Gómez y Limón, 1991 (como se citó en Campanario, 1997).

La implementación de elementos tecnológicos tales como calculadoras, computadores y dispositivos electrónicos resultan esenciales al momento de enseñar y aprender ciencias, y en particular la matemática, al mismo tiempo que ofrecen la oportunidad de obtener imágenes visuales de ideas matemáticas facilitando la organización y el análisis de los datos y la realización de cálculos en forma eficiente y exacta. De acuerdo con Santos (2000) Vega, et. al. (2015) la tecnología, como recurso de exploración y visualización, debe permitir que el estudiante establezca relaciones entre los distintos objetos matemáticos y se familiarice con las propiedades que estos cumplen, haciéndolos tangibles y manipulables en lugar de abstractos e imperceptibles.

La Universidad en Colombia y en general en el mundo, ha sufrido grandes transformaciones que tienen como hilo conductor la necesidad de adaptar los procesos formativos a las necesidades de una sociedad que cambia a una velocidad nunca antes conocida. En ese proceso de transformación, las tecnologías digitales juegan un papel importante y representan una verdadera transformación en los procesos de gestión, administración, investigación y docencia en la universidad (Marcelo, C., \& Rijo, D., 2019).

En este orden de ideas se plantea la siguiente pregunta de investigación ¿Cuál es el efecto de la implementación del software WINPLOT para el aprendizaje de la función lineal en estudiantes del primer semestre de la Facultad de Ciencias Empresariales y Económicas de la Universidad del Magdalena?

El abordaje de esta temática en particular está dado por dos aspectos centrales. Por un lado los bajos resultados académicos obtenidos por los estudiantes que cursaron la asignatura cálculo diferencial y que significó, para algunos, quedar en condición FBRA (fuera de la universidad por bajo rendimiento académico); y dos, la búsqueda de estrategias que permitan un mejor aprendizaje de la asignatura; entre ellas la integración de las tecnologías digitales por parte de los docentes que permitan el tránsito de una enseñanza del sentido común, de lo que siempre se ha hecho, hacia una docencia soportada en estrategias digitales. En este sentido, se espera que en sus procesos de aprendizaje, los estudiantes sean activos usuarios de las tecnologías digitales, asumiendo que "es posible concretar un entorno tecnológico donde el sujeto activa y sostiene 
su proceso de autorregulación del aprendizaje" (Chaves, Trujillo, \& López, 2016)

Duval (1988); Guzmán y Consigliere (1992); García, Vázquez e Hinojosa (2004) dan cuenta de las dificultades que se les presentan a los estudiantes de carreras del área de ingeniería, y de otras ciencias para la adquisición del concepto de función. Posiblemente esto se deba a la variedad de conceptos relacionados con éste, los cuales deben articularse para su mejor comprensión. Además, uno de los elementos intervinientes y con gran dificultad para aplicar son las operaciones algebraicas que permiten determinar dominios, rangos, intervalos de monotonía, entre otros, los cuales son de vital importancia en el desarrollo de un curso regular de Cálculo Diferencial.

Desde el punto de vista teórico, esta investigación sugiere que el uso del software gratuito WINPLOT, podría posibilitar la superación de dificultades propias del estudiante en relación con el aprendizaje de la función lineal. Según Riveros (2011), el uso de la tecnología puede ayudar a los estudiantes a aprender matemáticas, posibilita el acceso a modelos visuales, así como la capacidad para ampliar el rango de los problemas a los que puede acceder, $y$ fomenta el nivel de compromiso y apropiación de ideas matemáticas abstractas, pues las tecnologías de la información y la comunicación brindan tal versatilidad que otros medios no pueden ofrecer.

En este mismo sentido, los nuevos avances en la tecnología tienen muchas potencialidades para ser usadas en la formación matemática y científica de los estudiantes, y ello como respuesta a las dificultades atávicas que se experimentan en los procesos de aprendizaje y enseñanza de las matemáticas, que de acuerdo con Rico (1997) se localizan principalmente en:

- Dificultades asociadas a la complejidad de los objetos de las matemáticas.
- Dificultades asociadas a los procesos de pensamiento matemático.

- Dificultades asociadas a los procesos de enseñanza desarrollados para el aprendizaje de las matemáticas.

- Actitudes asociadas a los procesos de desarrollo cognitivo de los alumnos.

- Dificultades asociadas a actitudes afectivas y emocionales hacia las matemáticas.

Además de:

- Descontextualización y abstracción de los contenidos.

- $\quad$ Utilización de un lenguaje formal.

- Valoración del producto, ignorando el proceso seguido.

- Metodología deductiva, instructiva, repetitiva, en abandono de creatividad y la originalidad.

En respuesta a estas valoraciones, Ramírez (2015) encuentra que el aprendizaje de conceptos como el de función, operaciones con funciones y límites, se hace mucho más efectivo cuando el estudiante puede, a través de este programa, no solo visualizar el concepto como tal por medio de la graficación de funciones, sino también manipular estos objetos para entender de manera dinámica cómo los aspectos algebraicos generan transformaciones al objeto gráfico asociado y así entender de manera más precisa dichas transformaciones, no sólo desde la perspectiva del profesor, sino también desde su propia experiencia al permitírsele variar de manera voluntaria

En este sentido, Dunham (1993), reporta que muchos estudiantes que usan la tecnología de las calculadoras están mejor capacitados para: Relacionar las gráficas con sus ecuaciones, leer, interpretar información 
gráfica, obtener más información de las gráficas, simbolizar comprender mejor las conexiones entre representaciones gráficas, numéricas y algebraicas; además, exhiben estrategias flexibles ante la resolución de problemas, demuestran mayor inclinación hacia la resolución de tareas complejas, se concentran más en el problema y no en la manipulación algebraica, resuelven problemas no rutinarios que no se pueden resolver por técnicas algebraicas.

Bajo este entendido, se desarrolló este estudio, que corresponde a una investigación cuasi experimental de enfoque cuantitativo, tipo pre-test-pos-test con dos grupos, (experimental y control), de 15 estudiantes cada uno a los cuales se les aplicó dos pruebas de conocimiento sobre la temática función lineal. A los instrumentos se le aplicó prueba de validez a través de juicio de expertos y se utilizó la prueba alfa de Cronbach para determinar su confiabilidad.

\section{OBJETIVO GENERAL}

Determinar el efecto de la implementación del software WINPLOT para el aprendizaje de la función lineal en estudiantes del primer semestre de la Facultad de Ciencias Empresariales y Económicas de la Universidad del Magdalena.

\section{OBJETIVOS ESPECÍFICOS}

Determinar en los grupos experimental y control los resultados de aprendizaje de la función lineal antes y despues del tratamiento.

Determinar si existen diferencias entre los grupos experimental y control, en torno al aprendizaje de la función lineal

\section{DISEÑO DE INVESTIGACIÓN}

La presente investigación corresponde a un estudio enmarcado en el paradigma positivista de enfoque cuantitativo y diseño cuasiexperimental tipo pretest, postest. La investigación contó con un grupo experimental y otro de control con mediciones antes y después de la implementación del software Winplot en el grupo experimental, para evaluar el efecto del programa, en el aprendizaje de la función lineal. Después de concluido el período experimental, a ambos grupos se les aplicó simultáneamente una prueba (postest) relacionada con la función lineal, la cual fue equivalente al pretest. Se procedió inicialmente a la aplicación de la prueba "t" como prueba estadística para comparar los grupos, Esto permitió evaluar si difieren entre sí de manera significativa respecto a sus medias, y posteriormente se aplicó la prueba de correlación de Pearson y la regresión lineal, para determinar si existe correlación causal entre las variables.

\section{SISTEMA DE VARIABLES}

En la presente propuesta se considera como Variable independiente, la implementación del software WINPLOT y como Variable dependiente el Aprendizaje de la función lineal en estudiantes del primer semestre de la Facultad de Ciencias Empresariales y Económicas de la Universidad del Magdalena.

\section{RESULTADOS}

Se aplicó el procedimiento denominado prueba de hipótesis, definiendo la hipótesis nula: y alterna

: No existe una diferencia significativa entre la media de calificaciones del grupo control y la media de calificaciones del grupo experimental.

: Existe una diferencia significativa entre la media de calificaciones del grupo control y la media de calificaciones del grupo experimental.

Para la prueba estadística se utilizó un nivel de significancia del $5 \%$, es decir, . 
Como es un estudio de tipo transversal; porque se están analizando dos grupos (control y experimental) en un mismo momento con la misma prueba (pretest) y la variable aleatoria es numérica; pues se trata de las calificaciones obtenidas, se utilizará la prueba $t$ de Student para muestras independientes.

Los datos del pretest y el postest, se "corrieron utilizando el software SPSS para corroborar que la variable numérica aleatoria calificación se comporta normalmente y probar el supuesto de la igualdad de varianzas.
Los datos indican que la media de las calificaciones en el grupo experimental es ligeramente mayor que la del grupo control: 32,0833 y 30,4167 respectivamente. Sin embargo, se requiere precisar si la diferencia de medias es, o no significativa para determinar la homogeneidad o no de los grupos.

Se aplicó la prueba de normalidad obteniendo los siguientes resultados:

Tabla 1. Conocimientos previos. Pruebas de normalidad

\begin{tabular}{lc|c|c|c|c|c|}
\hline \multicolumn{8}{c|}{ Pruebas de Normalidad } \\
\hline \multirow{3}{*}{ Grupos } & \multicolumn{3}{c|}{ Kolmogorov-Smirnov } \\
\cline { 6 - 8 } & Valor Estadístico & GI & Sig. & Valor Estadístico & GI & Sig. \\
\cline { 2 - 4 } Control & 0,111 & 15 &, $200^{*}$ & 0,962 & 15 & 0,73 \\
Experimental & 0,141 & 15 &, $200^{*}$ & 0,935 & 15 & 0,33 \\
\hline
\end{tabular}

Fuente: Los autores

La lectura del P - Valor indica que en ambos grupos la variable calificación se comporta normalmente. (Tabla 2)

Tabla 2. Supuesto normalidad de calificaciones

\begin{tabular}{ccc}
\hline Normalidad de calificaciones \\
\hline$P-$ Valor (Grupo Control) $=0,734$ & $>$ & $\alpha=0,05$ \\
$P-\operatorname{Valor}(G r u p o$ Experimental) $=0,327$ & $>$ & $\alpha=0,05$ \\
\hline
\end{tabular}

Fuente: los autores

Para el supuesto de igualdad de varianzas, se observa, que el nivel de significancia arrojado por la prueba de Levene es de 0,971, el cual es mayor que $\alpha=0,05$. (Tabla 3 ), lo cual permite inferir que las varianzas de la variable calificación, son iguales para conocimientos previos en ambos grupos.
Es decir, estos datos permiten concluir que la variable calificación cumple con los dos supuestos: el de normalidad y el de igualdad de varianzas. 
Tabla 3. Prueba de muestras independientes

\begin{tabular}{|c|c|c|c|c|c|c|c|c|c|}
\hline & $\begin{array}{r}\text { Pr } \\
\text { Le } \\
\text { igu } \\
\text { va }\end{array}$ & $\begin{array}{l}\text { a de } \\
\text { e de } \\
\text { d de } \\
\text { izas }\end{array}$ & \multicolumn{7}{|c|}{ Prueba t para la igualdad de medias } \\
\hline & \multirow[t]{2}{*}{$\mathrm{F}$} & \multirow[t]{2}{*}{ Sig. } & \multirow[t]{2}{*}{$\mathrm{t}$} & \multirow[t]{2}{*}{$\mathrm{Gl}$} & \multirow[t]{2}{*}{$\begin{array}{c}\text { Sig. } \\
\text { (bilateral) }\end{array}$} & \multirow[t]{2}{*}{$\begin{array}{l}\text { Diferencia de } \\
\text { medias }\end{array}$} & \multirow[t]{2}{*}{$\begin{array}{l}\text { Diferencia } \\
\text { de error } \\
\text { estándar }\end{array}$} & \multicolumn{2}{|c|}{$\begin{array}{c}\text { 95\% de intervalo } \\
\text { de confianza de la } \\
\text { diferencia }\end{array}$} \\
\hline & & & & & & & & Inferior & Superior \\
\hline $\begin{array}{l}\text { Se asumen } \\
\text { varianzas } \\
\text { iguales }\end{array}$ & ,001 & ,971 &,- 295 & 28 & ,770 & $-1,66667$ & 5,647 & $-13,2351$ & 9,90182 \\
\hline $\begin{array}{l}\text { No se } \\
\text { asumen } \\
\text { varianzas } \\
\text { iguales }\end{array}$ & & &,- 295 & 27,985 & ,770 & $-1,66667$ & 5,647 & $-13,2354$ & 9,90210 \\
\hline
\end{tabular}

Fuente: los autores

Además, el nivel de significancia arrojado en la prueba $t$ de Student para la igualdad de medias es de 0,770, el cual es también mayor que $\alpha=0,05$. En consecuencia, no existe una diferencia significativa entre las medias de calificaciones del grupo control y el experimental, por lo que no hay evidencia para rechazar la hipótesis nula.

En consecuencia, se puede afirmar que los grupos presentan homogeneidad lo que permitió proceder con la implementación de la intervención.

Con el grupo experimental se desarrollaron las clases para la enseñanza de la función lineal utilizando el software Winplot, mientras que en el grupo control se desarrollaron en forma magistral sin ninguna ayuda tecnológica. Durante las clases los estudiantes del grupo experimental interactuaban con el software realizando mayor cantidad de ejercicios relacionados con los subtemas: intercepto con los ejes, dominio, rango y pendiente, entre otros.

Los datos de la postest, se "corrieron" utilizando el software SPSS para corroborar que la variable numérica aleatoria calificación se comporta normalmente y probar el supuesto de la igualdad de varianzas.

Los datos indican que la media de las calificaciones en el grupo experimental supera en 17,5 puntos a la del grupo control: 63,75 y 46,25 respectivamente. Sin embargo, se requiere precisar si la diferencia de medias es o no significativa, para determinar la homogeneidad o no de los grupos.

Se aplicó la prueba de normalidad la cual arrojó los siguientes resultados:

Tabla 4. Postest. Pruebas de normalidad

\begin{tabular}{llll|l|l|l|l|}
\hline \multicolumn{8}{c|}{ Pruebas de normalidad } \\
\hline \multirow{2}{*}{ Grupos } & Kolmogorov-Smirnov ${ }^{\mathrm{a}}$ Shapiro-Wilk \\
\cline { 3 - 8 } & Estadístico & gl & Sig. & Estadístico & gl & Sig. \\
\hline Control Después & 0,159 & 15 &, $200^{*}$ & 0,941 & 15 & 0,391 \\
Experimental Después & 0,162 & 15 &, $200^{*}$ & 0,952 & 15 & 0,560 \\
\hline
\end{tabular}

Fuente: los autores 
Los resultados anteriores muestran que la variable calificación en los grupos control y experimental se comportan normalmente, pues el grado o nivel de significancia de la variable calificación en ambos grupos es mayor que alfa. (Tabla 4).

La lectura del P - Valor indica que en ambos grupos la variable calificación se comporta normalmente. (Tabla 5)

Tabla 5. Supuesto normalidad de calificaciones

\begin{tabular}{ccc}
\hline \multicolumn{3}{c}{ Normalidad de calificaciones } \\
\hline$P-$ Valor (Grupo Control) $=0,391$ & $>$ & $\alpha=0,05$ \\
$P-$ Valor (Grupo Experimental) $=0,560$ & $>$ & $\alpha=0,05$ \\
\hline
\end{tabular}

Fuente: los autores

Para el supuesto de igualdad de varianzas, se observa, que el nivel de significancia arrojado por la prueba de Levene es de 0,079 , el cual es mayor que $\alpha=0,05$. (Tabla $6)$, lo cual permite inferir que las varianzas de la variable calificación, son iguales para el postest.
Es decir, estos datos permiten concluir que la variable calificación cumple con los dos supuestos: el de normalidad y el de igualdad de varianzas.

Tabla 6. Postest. Prueba de muestras independientes

\begin{tabular}{|c|c|c|c|c|c|c|c|c|c|}
\hline & \multicolumn{2}{|c|}{$\begin{array}{c}\text { Prueba de Levene } \\
\text { de igualdad de } \\
\text { varianzas }\end{array}$} & \multicolumn{7}{|c|}{ Prueba t para la igualdad de medias } \\
\hline & \multirow[t]{2}{*}{$\mathrm{F}$} & \multirow[t]{2}{*}{ Sig. } & \multirow[t]{2}{*}{$\mathrm{t}$} & \multirow[t]{2}{*}{ GI } & \multirow[t]{2}{*}{$\begin{array}{c}\text { Sig. } \\
\text { (bilateral) }\end{array}$} & \multirow[t]{2}{*}{$\begin{array}{c}\text { Diferencia de } \\
\text { medias }\end{array}$} & \multirow[t]{2}{*}{$\begin{array}{l}\text { Diferencia } \\
\text { de error } \\
\text { estándar }\end{array}$} & \multicolumn{2}{|c|}{$\begin{array}{c}\text { 95\% de intervalo } \\
\text { de confianza de la } \\
\text { diferencia }\end{array}$} \\
\hline & & & & & & & & Inferior & Superior \\
\hline $\begin{array}{l}\text { Se asumen } \\
\text { varianzas } \\
\text { iguales }\end{array}$ & 3,325 & 0,079 & $-2,43$ & 28 & 0,022 & $-17,5$ & 7,21688 & $-32,283$ & $-2,717$ \\
\hline $\begin{array}{l}\text { No se } \\
\text { asumen } \\
\text { varianzas } \\
\text { iguales }\end{array}$ & & & $-2,43$ & 25,01 & 0,023 & $-17,5$ & 7,21688 & $-32,363$ & $-2,637$ \\
\hline
\end{tabular}

Fuente: los autores

De otra parte, el nivel de significancia arrojado en la prueba t de Student para la igualdad de medias es de 0,022 , el cual es menor que $\alpha$ $=0,05$. En consecuencia, existe una diferencia significativa entre las medias de calificaciones del grupo control y el experimental, por lo que hay evidencia estadística para rechazar la hipótesis nula y asumir la hipótesis alterna

: Existe una diferencia significativa entre la media de calificaciones del grupo control y la media de calificaciones del grupo experimental. 
No obstante, se hace necesario estimar el efecto del software WINPLOT en el grupo experimental de estudiantes por cuanto partimos de la premisa de que existirá una diferencia significativa entre la media de calificaciones de los estudiantes en el grupo experimental antes del experimento y la media de calificaciones después del experimento. Por ello se plantearon las siguientes hipótesis:

Hipótesis Nula: No existe una diferencia significativa entre la media de calificaciones del grupo experimental antes del experimento y la media de calificaciones del grupo experimental después del experimento.

Hipótesis Alterna: Existe una diferencia significativa entre la media de calificaciones del grupo experimental antes del experimento y la media de calificaciones del grupo experimental después del experimento.

Para la prueba estadística se utilizó un nivel de significancia del $5 \%$, es decir, .

Tabla 7. Resultados de aprendizaje. Pretest, postest (medias)

\begin{tabular}{lcc}
\hline & Estadístico & Valor \\
\hline Pretest & Media & 32,0833 \\
Postest & Media & 63.75 \\
\hline
\end{tabular}

Fuente: los autores

De lo que se trata es de comparar el mismo grupo (experimental) en dos momentos temporalmente distintos, pues se trata de las calificaciones obtenidas en el pretest y en la postest; para ello se utilizó la prueba $t$ de Student para muestras relacionadas.

Antes de determinar el nivel de significancia, se corroboró el supuesto de normalidad utilizando también, el SPSS.

Para este caso se encontró que la variable calificación en el grupo experimental, antes y después del experimento, se comportan normalmente, pues el nivel de significancia en los dos momentos es mayor que el valor de alfa (Tabla 8).

Tabla 8. Grupo experimental. Pruebas de normalidad

\begin{tabular}{|c|c|c|c|c|c|c|}
\hline \multicolumn{7}{|c|}{ Pruebas de normalidad } \\
\hline \multirow{2}{*}{ Grupos } & \multicolumn{3}{|c|}{ Kolmogorov-Smirnov ${ }^{a}$} & \multicolumn{3}{|c|}{ Shapiro-Wilk } \\
\hline & Estadístico & $\mathrm{Gl}$ & Sig. & Estadístico & $\mathrm{Gl}$ & Sig. \\
\hline Pretest & ,141 & 15 & ,200* & ,935 & 15 & ,327 \\
\hline Postest & ,162 & 15 & ,200* & ,952 & 15 & ,560 \\
\hline
\end{tabular}

Fuente: los autores

En la prueba $t$ para muestras relacionadas realizada, se encontró, que el nivel de significancia $(0,0000)$ es menor que $\alpha=0,05$, y el valor de la correlación de 0,868 (Tabla 9). Por consiguiente, se acepta la hipótesis alterna, pues existe diferencia significativa entre la media de calificaciones del grupo experimental, antes y después del experimento y las variables están altamente correlacionadas. Esta situación se corroboró con el cálculo del coeficiente de correlación de Pearson. Es decir, la variable independiente, implementación del software WINPLOT, esta altamente correlacionada con la variable dependiente, aprendizaje de la función 
lineal en estudiantes del primer semestre de la Facultad de Ciencias Empresariales y Económicas de la Universidad del Magdalena

Tabla 9. Correlaciones de muestras emparejadas

\begin{tabular}{llccc}
\hline & & N & Correlación & Sig. \\
\hline Par 1 & $\begin{array}{l}\text { Calificaciones pretest \& } \\
\text { Calificaciones postest }\end{array}$ & 15 &, 868 &, 000 \\
\hline
\end{tabular}

Fuente: los autores

Para determinar si existe correlación entre las variables, se determinó el coeficiente de correlación de Pearson y la regresión lineal para establecer si las variables en estudio están relacionadas existiendo entre ellas una correlación causal.

Para el primer caso, (Tabla 10) se encontró que el valor de la correlación es de y el nivel de significancia corresponde a 0,000; lo cual nos evidencia que existe una relación lineal directa entre la variable independiente implementación del software WINPLOT y la variable dependiente aprendizaje de la función lineal en estudiantes del primer semestre de la Facultad de Ciencias Empresariales y Económicas de la Universidad del Magdalena y que además es fuerte puesto que dicho valor se acerca a 1.

Tabla 10. Correlación de Pearson

\begin{tabular}{|l|c|c|c|}
\hline & & $\begin{array}{c}\text { Calificaciones } \\
\text { Postest }\end{array}$ & $\begin{array}{c}\text { Calificaciones } \\
\text { Pretest }\end{array}$ \\
\hline \multirow{3}{*}{$\begin{array}{l}\text { Calificaciones } \\
\text { postest }\end{array}$} & $\begin{array}{c}\text { Correlación de } \\
\text { Pearson }\end{array}$ & 1 &, $868^{* *}$ \\
\cline { 2 - 4 } & Sig. (bilateral) & & 0,000 \\
\cline { 2 - 4 } & $\mathrm{N}$ & 15 & 15 \\
\hline \multirow{3}{*}{$\begin{array}{l}\text { Calificaciones } \\
\text { Pretest }\end{array}$} & $\begin{array}{c}\text { Correlación de } \\
\text { Pearson }\end{array}$ &, $868^{* *}$ & 1 \\
\cline { 2 - 4 } & Sig. (bilateral) & 0 & 15 \\
\cline { 2 - 5 } & $\mathrm{N}$ & 15 & \\
\hline \multirow{2}{*}{ **. La correlación es significativa en el nivel 0,01 (bilateral). } & \\
\hline
\end{tabular}

Fuente: los autores

Para el segundo caso (Figura 1), se observa que la mayor cantidad de puntos se encuentra ubicados en el primer y tercer cuadrante, los cuales aportan un valor positivo a la covarianza, indicando que su valor es mayor que cero. Esta característica evidencia una relación directa entre las variables objeto de estudio en esta investigación la cual corresponde a una correlación causal. 
Figura 1. Signo de la covarianza

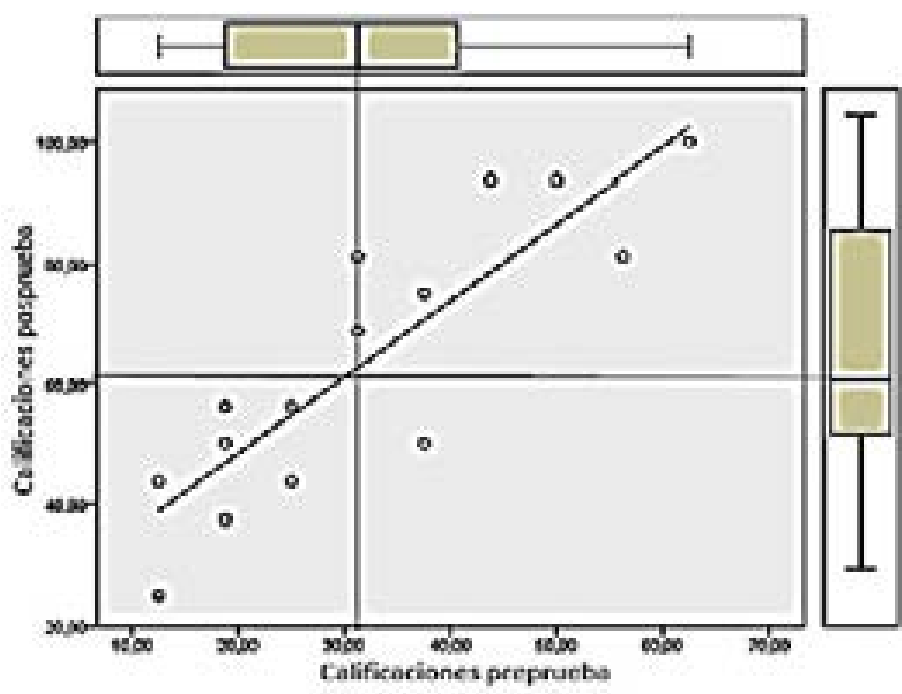

Fuente: los autores

Se evidencia que para todos los estudiantes del grupo experimental la calificación después del tratamiento siempre es mayor que la calificación antes del tratamiento, pues si trazamos una línea de 45 grados de inclinación, todos los puntos se encuentran por encima de dicha línea.

Teniendo en cuenta el análisis realizado anteriormente, se puede afirmar que después de la implementación del software WINPLOT en el aprendizaje de la función lineal en estudiantes de primer semestre de la Facultad de Ciencias Empresariales y Económicas de la Universidad del Magdalena al grupo experimental, los resultados obtenidos por los estudiantes en la postest mejoraron de manera significativa. Además, se observó una participación más activa, solución de mayor número de ejercicios en cada uno de los subtemas abordados (intercepto con los ejes, dominio, rango y pendiente, entre otros).

Lo anterior es evidencia de que la utilización del software WINPLOT, constituye una propuesta pedagógica que contribuye a mejorar las dificultades que presentan los estudiantes en el tema de la función lineal, como lo evidenció Colmenares (2011), Borba (1995b), quien señala que representaciones múltiples, favorecidas por algunos software matemáticos, pueden desafiar el monopolio de las expresiones algebraicas en la educación matemática sin perder de vista la importancia del uso de estas expresiones" ( $p$. 89).

\section{CONCLUSIONES}

Un aspecto para destacar, lo constituye la tasa de reprobación de la asignatura Cálculo Diferencial en la Facultad de Ciencias Empresariales y Económicas de la Universidad del Magdalena, la cual se aproxima al $60.1 \%$. Esta situación, siempre se ha explicado a partir de la "mala preparación" con que llegan los estudiantes a la Universidad, así como al bajo dominio de competencias lógico matemáticas; es decir, asociadas al alumno y nunca, para el caso de nuestro contexto, se ha vislumbrado la posibilidad de asumir una posible causa, asociada a las estrategias didácticas utilizadas por los docentes en el proceso de enseñanza. 
Aunque el concepto de rendimiento académico es polisémico, en esta investigación lo asumimos desde la perspectiva planteada por Touron (1985), quien lo concibe como "el resultado del aprendizaje suscitado por la actividad educativa del profesor y producido en el alumno, aunque es claro que no todo aprendizaje es fruto exclusivo de la acción docente"

Se entiende que el desempeño académico de los estudiantes, se debe a múltiples causas, no obstante, las asociadas al estudiante y al docente constituyen, tal vez, las de mayor relevancia, por lo que corresponden a la interacción docente-alumno durante los procesos de enseñanza y aprendizaje. Esto no significa que no se conjuguen otras razones para la perdida de la asignatura, asociadas a situaciones laborales, familiares, o de otro tipo, sin embargo, se decide abordar esta investigación partiendo de la idea que si se utilizan estrategias basadas en las TIC, es posible que se presente un mejor aprendizaje.

La implementación del software WINPLOT para el aprendizaje de la función lineal en estudiantes del primer semestre de la Facultad de Ciencias Empresariales y Económicas de la Universidad del Magdalena, evidenció que:

Los resultados de aprendizaje obtenidos por los estudiantes pertenecientes al grupo experimental en el tema de la función, lineal después de la implementación del software WINPLOT, evidencian que se presentó una mejora significativa en sus calificaciones con relación a las obtenidas antes del experimento, pudiendo afirmarse que con la implementación de dicho software se favorece de manera positiva el aprendizaje de los estudiantes.

Existe una correlación (fuerte) causal entre la variable independiente implementación del software WINPLOT y la variable dependiente aprendizaje de la función lineal en estudiantes del primer semestre de la Facultad de Ciencias Empresariales y Económicas de la Universidad del Magdalena.

La utilización del software WINPLOT, constituye una estrategia didáctica que contribuye a mejorar el aprendizaje de los estudiantes en el tema de la función lineal.

Estos resultados corroboran los de otras investigaciones, verbigracia, Dunham y Dick (1994); Sheets (1993); Boers-van Oosterum (1990); Rojano (1996); Groves (1994), que dan cuenta de que los estudiantes pueden aprender más matemáticas y con mayor profundidad mediante el uso apropiado de la tecnología. Sin embargo, la tecnología no se debe utilizar como un reemplazo de la comprensión básica y de las intuiciones; más bien, puede y debe utilizarse para fomentar las comprensiones e intuiciones de conceptos matemáticos y con frecuencia y responsabilidad, con el objeto de enriquecer el aprendizaje de los estudiantes.

Desde el punto de vista de la factibilidad, las diferentes aplicaciones tecnológicas se convierten entonces en una herramienta para la enseñanza, las cuales utilizadas de forma adecuada permitirán mejorar los procesos de aprendizaje de los estudiantes en relación con el tema de la función lineal, así como la posibilidad de examinar otros ejemplos o representaciones que no son posible realizar manualmente, y que fácilmente pueden realizar exploraciones y hacer conjeturas. A medida que los estudiantes utilizan el WINPLOT en el computador, se tiene la oportunidad de observarlos y apreciar como razonan, lo cual es difícil de observar en otras circunstancias.

No cabe duda, que las herramientas tecnológicas han sido de gran utilidad en la educación, y además su uso apropiado corresponde a uno de los principios formulados por el Consejo Estadounidense de Profesores 
de Matemáticas NCTM (2000), el cual establece que la tecnología es fundamental en la enseñanza y el aprendizaje de las matemáticas, e influye en su enseñanza y mejora el proceso de aprendizaje, implicando según Pastran, Gil y Cervantes (2020) "la transformación de los roles tradicionales de docentes y estudiantes en papeles más dinámicos e interactivos".

Así mismo, tecnologías electrónicas como calculadoras y computadores, constituyen imprescindibles herramientas para enseñar, aprender y "hacer" matemáticas y ofrecen imágenes visuales de ideas matemáticas, facilitando la organización y el análisis de los datos y la realización de cálculos en forma eficiente y exacta. Sin embargo, como este uso no ha sido generalizado, por lo menos en el país, se hace necesario un mayor acompañamiento para los docentes en cuanto al manejo de herramientas TICS, pues los estudiantes tienen mucha destreza en el manejo de ellas por lo que resulta importante incluirlos en los procesos de aprendizaje. (Ahumada, D., 2021).

\section{REFERENCIAS BIBLIOGRÁFICAS}

Ahumada Ebratt, D. D. (2021). El uso TIC'S en el proceso de enseñanza-aprendizaje del alemán como lengua extranjera en la universidad. Revista Boletín Redipe, 10(5), 248-258. https://doi.org/10.36260/ rbr.v10i5.1301

Aguilera, M.N., y Jiménez, V.E. (2012). Factores de deserción universitaria en el primer curso de las carreras de Trabajo Social y lengua inglesa en las Facultades de Humanidades y Ciencias de la Educación y de lenguas Vivas de la Universidad Evangélica de Paraguay. Revista Internacional de Investigación en Ciencias Sociales, 8(2), 197-205.
Alzás, T., y Casas, L. (2015). Relato autobiográfico del abandono educativo. Una visión integrada desde metodologías mixtas. Investigación Cualitativa en Ciencias Sociales, 3, 108-113

Belloc, F., Maruotti, A., y Petrella, L. (2011). How individual characteristics affect university students drop-out: a semiparamentric mixed-effects model for an Italian case study. Journal of Applied Statistics, 38(10), 2225-2239. 10.1080/02664763.2010.545373

Bernardo, A., Cervero, A., Esteban, M., Fernández, E., y Núñez, J.C. (2016). Influencia de variables relacionales y de integración social en la decisión de abandonar los estudios en Educación Superior. Psicologia, Educação e Cultura, $X X(1), 138-151$.

Boers-Van Oosterum, M. A. M. (1990). Understanding of variable and their uses acquired by students in traditional and computer-intensive algebra [doctoral dissertation, University of Maryland College Park]. Dissertation abstracts International. https://dl.acm.org/doi/ book/10.5555/916801

Borba, M. (1995b), "Funções, representações múltiplas e visualização na Educação Matemática" [ponencia]. I Seminário Internacional de Educação Matemática, Rio de Janeiro, Brasil.

Bozick, R. (2007). Making it through the first year of college: the role of students' economic resources, employment, and living arrangements. Sociology of Education, 80(3), 261-285. $10.1177 / 003804070708000304$ 
Calatayud, M.L., Gil, D. y Gimeno, J.V. (1992). Cuestionando el pensamiento docente espontáneo del profesorado universitario: ¿Las deficiencias en la enseñanza como origen de las dificultades de los estudiantes? Revista Interuniversitaria de Formación del Profesorado, 14(2), 71-81. https://dialnet.unirioja.es/servlet/ articulo?codigo $=254985$

Carrascosa, J. y Gil, D. (1985). La «metodología de la superficialidad» y el aprendizaje de las ciencias. Enseñanza de las Ciencias, $3,113-120$.

Castejón, A., Ruiz, M., Arriaga, J., y Casaravilla, A. (2016). Modelo estructural causal de la permanencia en la Universidad Politécnica de Madrid. Sexta Conferencia Latinoamericana sobre el Abandono en Educación Superior (VI CLABES). Quito, Ecuador.

Colmenares, G. (2011). Herramientas Computacionales como Estrategia para el Aprendizaje de Relaciones y Funciones Matematicas en Educación Media en General [tesis de maestría/no publicada, Universidad del Zulia].

Consejo Estadounidense de Profesores de Matemáticas. (2000). El principio de la tecnología para matemáticas escolares. (Documento del NCTM). http://eduteka. icesi.edu.co/articulos/PrincipiosMath

Chaves, E., Trujillo, J.M. y López, J.A. (2016). Acciones para la autorregulación del aprendizaje en entornos personales, España. Revista de Medios y Educación, 48, 67-82. https://www.redalyc.org/ pdf/368/36843409006.pdf
Dunham, P. y Dick, T. (1994). Research on graphic calculators. The Mathematics Teacher, 87(6), 440-445. https://search. proquest.com/docview/204609004?pqorigsite $=$ gscholar

Dunham, P. (1993). "Does using calculators work. The jury is almost in". UME Trends, 5(2), 8-9.

Duval, R. (1988). Graphiques et équations: l'articulation de deux registres. Annales de Didactique et de Sciences Cognitives, 1, 235-253. http://numerisation.irem.univ$\underline{\mathrm{mrs} . f r / S T / / S T 88014 / I S T 88014 . p d f}$

Elías, M. (2008). Los abandonos universitarios: retos ante el Espacio Europeo de Educación Superior. Estudios sobre Educación, 15, 101-121.

Feixas, M., Muñóz, J.L., Gairín, J., RodríguezGómez, D., y Navarro, M. (2015). Hacia la comprensión del abandono universitario en Catalunya: el caso de la Universitat Autònoma de Barcelona. Estudios sobre Educación, 28, 117-138. 10.15581/004.28.117-138

García, A., y Adrogué, C. (2015). Abandono de los estudios universitarios: dimensión, factores asociados y desafíos de la política pública. Revista Fuentes, 16, 85-106. http://dx.doi.org/10.12795/ revistafuentes.2015.i16.04

García, J.J. y Cañal, P. (1995). ¿Cómo enseñar? Hacia una definición de las estrategias de enseñanza por investigación. Investigación en la Escuela, 25, 7. https:// idus.us.es/handle/11441/59627

García-Quiroga, L, Vázquez-Cedeño, R. y Hinojosa-Rivera, M. (2004). Dificultades en el aprendizaje del concepto de función 
en estudiantes de ingeniería. Ingenierías, 7(24), 27-34. http://eprints.uanl.mx/10145/

Goldenhersh, H., Coria, A., y Saino, M. (2011). Deserción estudiantil: desafíos de la universidad pública en un horizonte de inclusión. Revista Argentina de Educación Superior, 3(3), 96-120.

Groves, S. (1994, April 4-8). Calculators: A learning environment to promote number sense [paper]. Annual meeting of the American Educational Research Association, New Orleans. https://eric. ed.gov/?id=ED373969

Guzmán, I. y Consigliere, D. (1992). Algunas dificultades de aprendizaje detectadas en alumnos de Cálculo Diferencial. Revista de Educación Matemática, 4(1), 54-64. http://funes.uniandes.edu.co/9529/

Marcelo, C. (2001, 6-8 de junio). Rediseño de la práctica pedagógica: factores, condiciones y procesos de cambios en los teletransformadores [conferencia]. Reunión Técnica Internacional sobre el uso de TIC en el Nivel de Formación Superior Avanzada, Sevilla, España.

Marcelo, C., y Rijo, D. (2019). Aprendizaje autorregulado de estudiantes universitarios: Los usos de las tecnologías digitales. Revista Caribeña de Investigación Educativa (RECIE), 3(1), 62-81. https://doi.org/10.32541/ recie.2019.v3i1.pp62-81

Montero, E.; Villalobos, J., y Valverde, A. (2007). Factores institucionales, pedagógicos, psicosociales y sociodemográficos asociados al rendimiento académico en la Universidad de Costa Rica: Un análisis multinivel. Revista Electrónica de Investigación y Evaluación Educativa
(RELIEVE), 13(2): 215-234. https://doi. org/10.7203/relieve.13.2.4208

Pastran Chirinos, M., Gil Olivera, N. A., \& Cervantes Cerra, D. (2020). En tiempos de coronavirus: las TIC'S son una buena alternativa para la educación remota. Revista Boletín Redipe, 9(8), 158-165. https://doi.org/10.36260/rbr.v9i8.1048

Ramírez, C. (2015). Diseño de herramientas que fomentan el aprendizaje de matemáticas con ayuda de Mathematica 10. Revista Elementos, 5, 65-78. https://dialnet.unirioja. es/servlet/ articulo?codigo $=5179413$

Rico, L. (1997). La Educación Matemática en la enseñanza secundaria. Institut de Ciéncies de Éducació (ICE). Universitat de Barcelona. pp.125-154.

Riveros, V. (2011). Las tecnologías de la información y la comunicación en la construcción del conocimiento matemático. Algunos criterios teóricos. Encuentro Educacional. Revista especializada en Educación, 18(3), 418419.

Riveros, V. y Mendoza, M. (2008). "Consideraciones teóricas del Uso de Internet en educación". Revista OMNIA, 14(1), 27-46.

Rojano, T. (1996). Developing Algebraic Aspects of Problem Solving Within a Spreadsheet Environment. En N. Bednarz, L. Lee y C. Kieran (eds.), Approaches to Algebra: Perspectives for Research and Teaching. Londres-Boston, Inglaterra-Estados Unidos: Kluwer Academic Publishers. 
Rué, J. (2014). El abandono universitario: variables, marcos de referencia y políticas de calidad. Revista de Docencia Universitaria, 12(2), 281-306.

Sheets, C. (1993). Effects of computer learning and problem-solving tools on the development of secondary school students' understanding of mathematical functions [doctoral dissertation, University of Maryland, College Park] https://elibrary. $\underline{\text { ru/item } . a s p ? i d=5759402}$

Vega-Vega, J.C., Niño-Duarte, F. y Cárdena, Y.P. (2015). Enseñanza de las matemáticas básicas en un entorno e-Learning: un estudio de caso de la Universidad Manuela Beltrán Virtual. Revista Escuela de Administración de Negocios, 79, 172-185. http://www.scielo. org.co/scielo.php?script=sci_arttext\&pid $=$ S0120-81602015000200011

Wilson, B. (1996). Constructivist learning environment. New Jersey, USA: Educational Technology.

Tourón-Figueroa, J. (1985). "La predicción del rendimiento académico: Procedimientos, resultados e implicaciones". Revista Española de Pedagogía, 43(169170), 473-495. http://www. jstor.org/ stable/23764399

Tuero, E., Cervero, A., Esteban, M., y Bernardo,A. (2018). ¿Por qué abandonan los alumnos universitarios? Variables de influencia en el planteamiento y consolidación del abandono. Educación XX1, 21(2), 131154, doi: 10.5944/educXX1.20066 